\title{
DETECTION OF HAMILTONIAN CIRCUITS IN A DIRECTED GRAPH
}

\author{
SUDHANGSHU B. KARMAKAR'
}

(Received 26 March 1981; revised 22 December 1981)

\begin{abstract}
A simple algebraic method is presented to determine the necessary condition for the existence of a Hamiltonian circuit in a directed graph of $n$ vertices. A search procedure is then introduced to identify any or all of the existing Hamiltonian circuits. The procedure is based upon finding a set of edges which will then be candidates for being parts of circuits of length $n$ at any vertex of the graph.
\end{abstract}

\section{Introduction}

A circuit in a connected graph is said to be Hamiltonian if it includes every vertex once. Hence a Hamiltonian circuit in a graph of $n$ vertices consists of exactly $n$ edges. Because of its practical application the subject has generated renewed interest in diverse fields of engineering sciences since it was first proposed by the Irish mathematician Sir William Rowan Hamilton in 1895. A method for finding whether a graph contains a Hamiltonian circuit or not has direct applications in problems of sequencing or scheduling of operations. Equally important, however, is the use of such a method as a basic step in algorithms for the solution of other seemingly unrelated graph theory problems [3].

A Hamiltonian path [7] is closely related to a corresponding Hamiltonian circuit and is obtained by removing one edge from the circuit. Thus every graph that has a Hamiltonian circuit also has a Hamiltonian path; obviously the reverse is not necessarily true.

\footnotetext{
'Bell Laboratories, Room ID-291, Piscataway, New Jersey 08854, U.S.A.

(C) Copyright Australian Mathematical Society 1982
} 
The problem of determining Hamiltonian circuits in a given graph has offered an interesting challenge to the applied mathematicians and graph theorists. But in spite of the various attempts so far no efficient algorithm has been developed to characterize such a circuit in a graph. In fact it has been shown $[4,5]$ that the problem for finding a Hamiltonian circuit in a graph is one of $N P$ complete problems. Consequently, attention has been directed to the development of efficient algorithms for some special but useful cases. Some sufficient conditions for the existence of a Hamiltonian circuit have been obtained in terms of degree sequence of a graph [2]. Takamizawa et al. presented an $O\left(n^{3}\right)$ time algorithm for finding a Hamiltonian circuit in a diconnected graph satisfying Meyniel's condition [6].

This paper considers a general case and presents a simple algorithm to detêrmine the necessary condition for the existence of any Hamiltonian circuits in a directed graph. On the basis of the necessary condition a search procedure is then developed to detect and identify all the existing Hamiltonian circuits in the graph.

\section{Terminology}

Standard graph theory terminology has been used throughout. For the sake of clarity, the following terms are repeated.

A graph $G=(V, E)$ consists of a finite, nonempty set of vertices $V$ and a set of edges $E$. If the edges are ordered pairs $(u, w)$ of vertices, then the graph is said to be directed. The vertex $u$ is called the tail and $w$ the head of the edge $(u, w)$. If the edges are unordered pairs of distinct vertices then the graph is said to be undirected. The graph $G$ is assumed to have $n$ vertices. The degree of a vertex $u$, denoted by $d(u)$, is the number of edges incident to $u$.

Adjacency matrix. The adjacency matrix $A$ of a directed graph $G$ is an $n$ by $n$ matrix with an element $a_{i}$, such that

$$
a_{i j}= \begin{cases}1 & \text { if there is an edge from vertex } i \text { to vertex } j, \\ 0 & \text { otherwise. }\end{cases}
$$

Connection matrix. The connection matrix $C$ of the graph $G$ is a square matrix of order $n$ with an element $c_{i}$, and represents the simplified structure of $G$, that is, $c_{i j}=0$ if there is no edge from vertex $i$ to vertex $j$. Otherwise, $c_{i j}>0$ and indicates that there is at least one directed edge connecting vertex $i$ to vertex $j . c_{t i}$ is taken to be identically zero. That is, all the self loops in $G$ are ignored. 


\section{Symbols}

In addition to the above, additional symbols are introduced for ease of discussion. $A^{p}$ is the $p$ th power of the adjacency matrix $A$ and $a_{i j}^{(p)}$ is an element of the matrix $A^{p}, p=1,2, \ldots, n$. The matrix $\tilde{A}_{1}$ is obtained by zeroing all the diagonal elements of $A . \tilde{a}_{i j}^{(1)}$ is an element of $\tilde{A}_{1}$. The matrices $A_{k}$ and $\tilde{A}_{k}$, $k=2,3, \ldots, n$, are defined recursively as follows:

The operation ' $*$ ' is the same as the ordinary row by column multiplication in matrix theory except that ' + ' operation is done according to the following table:

\begin{tabular}{c|cc}
+ & 0 & 1 \\
\hline 0 & 0 & 1 \\
1 & 1 & 1
\end{tabular}

We define

$$
A_{2}=\bar{A}_{1} * \tilde{A}_{1} \text {. }
$$

$\tilde{A}_{2}$ is obtained by zeroing all the diagonal elements of $A_{2}$. Similarly,

$$
A_{3}=\tilde{A}_{2} * \tilde{A}_{1} \text {. }
$$

$\tilde{A}_{3}$ is obtained by zeroing all the diagonal elements of $A_{3}$. Continuing the process finally we get

$$
A_{k}=\tilde{A}_{k-1} * \tilde{A}_{1} \text {. }
$$

Let $\tilde{a}_{i j}^{(k)}$ be an element of $\tilde{A}_{k}$ and $\bar{a}_{1 j}^{(k)}$ be an element of $A_{k}$. Thus,

$$
\begin{array}{ll}
\tilde{a}_{i j}^{(k)}=\bar{a}_{i j}^{(k)} & \text { for } i \neq j, \\
\tilde{a}_{i j}^{(k)}=0 & \text { for } i=j .
\end{array}
$$

Clearly, $\tilde{a}_{i j}^{(k)}$ and $\tilde{a}_{i j}^{(k)}$ can assume values 0's and l's only.

\section{Development of the algorithm}

Since any loop at any one of the vertices will contribute nothing to the final determination of the Hamiltonian circuit, its effect is nullified by excluding it from the adjacency matrix. Thus, the starting matrix is taken to be $\tilde{A}_{1}$ not $A$.

Let any circuit of length $<n$ at any one of the vertices be called a local circuit. From the well known properties of the adjacency matrix [1], it is known that if $a_{i j}^{(p)}=q>0$ then the vertex $v$, can be reached from vertex $v$, in $p$ steps by $q$ different ways. Thus if $\bar{a}_{i t}^{(2)} \neq 0$, there will be a local circuit of length 2 at the vertex $v_{\imath}$. The contribution of this local circuit in the next matrix multiplication is 
nullified by replacing $A_{2}$ by $\tilde{A}_{2}$. Again if $\bar{a}_{u}^{(3)} \neq 0$, there will be a local circuit of length 3 at the vertex $v_{i}$. Its contribution in the next matrix multiplication is avoided by replacing $A_{3}$ by $\tilde{A}_{3}$. Continuing this process by ordinary matrix multiplication, define a detection matrix as:

$$
D_{n}=\tilde{A}_{n-1} \times C \text {. }
$$

Let $d_{i}$, be an element of the matrix $D_{n}$. Clearly if any one of $d_{i i}=0$ for $i=1,2, \ldots, n$, there cannot exist any circuit of length $n$ at the vertex $v_{t}$. Thus the necessary condition for the existence of any Hamiltonian circuit is that

$$
d_{u} \neq 0 \text { for all } i=1,2, \ldots, n \text {. }
$$

It is emphasized here that the multiplication of $\tilde{A}_{k}$ by $\tilde{A}_{1}$ is equivalent to adding an arc at the beginning of a path recorded by $\tilde{A}_{k}$. Thus even though $d_{u} \neq 0$, it does not necessarily indicate the existence of any Hamiltonian circuit; it only indicates that there is a circuit of length $n$. Thus the above zeroing method succeeds only in eliminating paths that end with a loop. The objective of zeroing the diagonal elements is to eliminate as many edges as possible which will not contribute in the final identification of the existing Hamiltonian circuits. When $d_{i i} \neq 0$, from (2)

$$
d_{i i}=c_{p i}+c_{q 1}+\cdots+c_{r i},
$$

where $p, q, r$, etc. are all distinct and $1 \leqslant p, q, \ldots, r \leqslant n$. The edges $c_{p l}, c_{q l}, \ldots, c_{r t}$ are all incident to the vertex $v_{t}$ and thus potential candidates for being parts of any existing Hamiltonian circuits. Let the number of edges incident on vertex $v_{l}$, as obtained from (3), be denoted by $t_{i}$. Thus $t_{i}$ can be viewed as the effective degree of the vertex $v_{\text {, }}$.

Now in order to trace the existing Hamiltonian circuits in the graph the concept of reachability is introduced as follows:

From (3), the vertex $v$, can be reached in one step from each of the vertices $v_{p}, v_{q}, \ldots, v_{r}$. The reachability of the vertex $v_{1}$ is given by

$$
v_{i} \leftarrow \sum_{j \neq i}^{t_{j} j}
$$

where $j$ can only assume values $p, q, \ldots, r$ as obtained from (3) and $t_{t}$ is the number of terms in the right hand side of (3).

Next pick up a vertex $v_{i}$ corresponding to minimum $t_{t}$. Write the reachability at vertex $v_{\text {. }}$. This will generate a series of vertices. Again apply reachability from (4) at each of the vertices. This process is repeated $n$ times. If in the process of any vertex is repeated, further processing at that vertex is discontinued. Hamiltonian circuits can now easily be traced by noting the starting and ending vertices of the circuit of length $n$. 


\section{Illustrative examples}

The following three examples are presented to illustrate the technique. Example 1. Consider the graph of Figure 1. Here

$$
\begin{gathered}
A=\left[\begin{array}{lllll}
0 & 1 & 1 & 0 & 0 \\
0 & 0 & 0 & 1 & 0 \\
1 & 1 & 1 & 0 & 0 \\
0 & 0 & 1 & 0 & 1 \\
1 & 1 & 1 & 1 & 0
\end{array}\right], \quad \tilde{A}_{1}=\left[\begin{array}{lllll}
0 & 1 & 1 & 0 & 0 \\
0 & 0 & 0 & 1 & 0 \\
1 & 1 & 0 & 0 & 0 \\
0 & 0 & 1 & 0 & 1 \\
1 & 1 & 1 & 1 & 0
\end{array}\right], \\
C=\left[\begin{array}{llllll}
0 & c_{12} & c_{13} & 0 & 0 \\
0 & 0 & 0 & c_{24} & 0 \\
c_{31} & c_{32} & 0 & 0 & 0 \\
0 & 0 & c_{43} & 0 & c_{45} \\
c_{51} & c_{52} & c_{53} & c_{54} & 0
\end{array}\right], \quad \tilde{A}_{4}=\left[\begin{array}{lllll}
0 & 1 & 1 & 1 & 1 \\
1 & 0 & 1 & 1 & 1 \\
1 & 1 & 0 & 1 & 1 \\
1 & 1 & 1 & 0 & 0 \\
1 & 1 & 1 & 1 & 0
\end{array}\right] .
\end{gathered}
$$

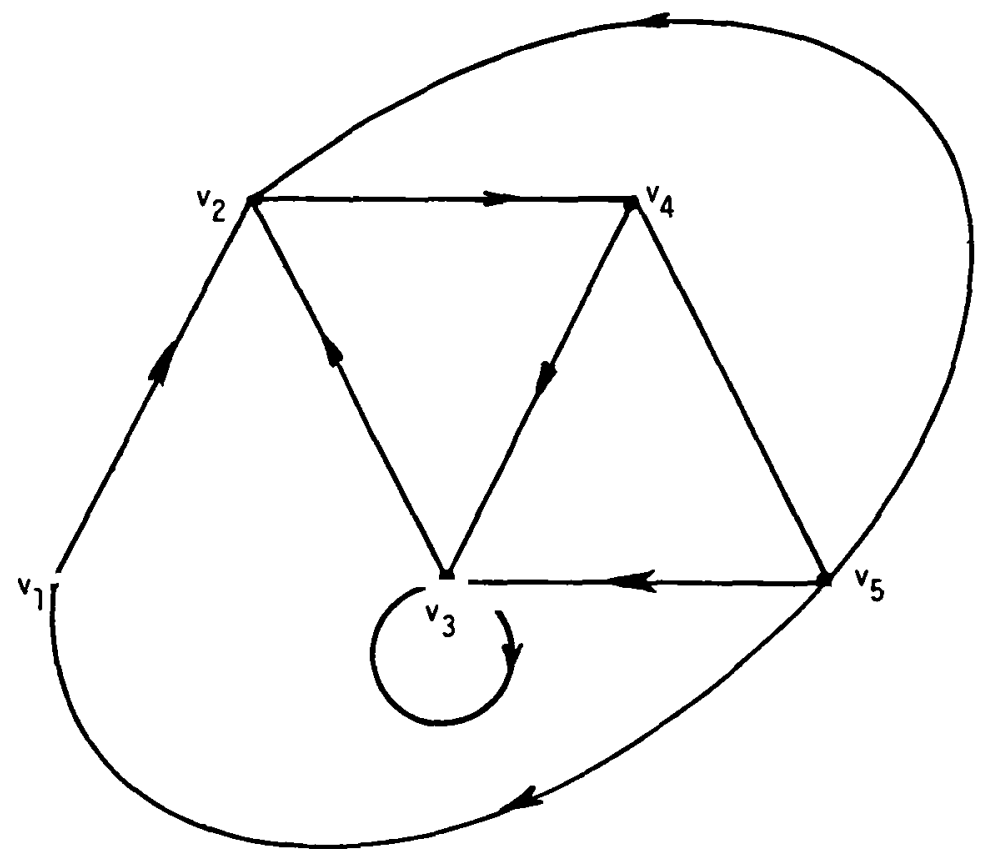

Figure 1. Graph of Example 1.

From (2),

$$
\begin{gathered}
d_{11}=c_{31}+c_{51}, \quad d_{22}=c_{12}+c_{32}+c_{52}, \\
d_{33}=c_{13}+c_{43}+c_{53}, \quad d_{44}=c_{24}, \quad d_{55}=c_{45} .
\end{gathered}
$$


Since $d_{i i} \neq 0$ for all $i=1,2, \ldots, 5$, the necessary condition for the existence of a Hamiltonian circuit is satisfied. In order to trace the circuit, the reachability at each of the vertices is written from (4) as shown below.

$$
\begin{gathered}
v_{1} \leftarrow 3+5, \quad v_{2} \leftarrow 1+3+5, \\
v_{3} \leftarrow 1+4+5, \quad v_{4} \leftarrow 2, \quad v_{5} \leftarrow 4 .
\end{gathered}
$$

Now starting with the vertex $v_{5}$ corresponding to $\min \left(t_{t}\right)=1$, write successively the reachability at each of the vertices thus generated. The whole process is shown in Figure 2. The following two circuits are detected.

(i) $t \rightarrow 3 \rightarrow 1 \rightarrow 2 \rightarrow 4 \rightarrow 5$,

(ii) $5 \rightarrow 1 \rightarrow 3 \rightarrow 2 \rightarrow 4 \rightarrow 5$.

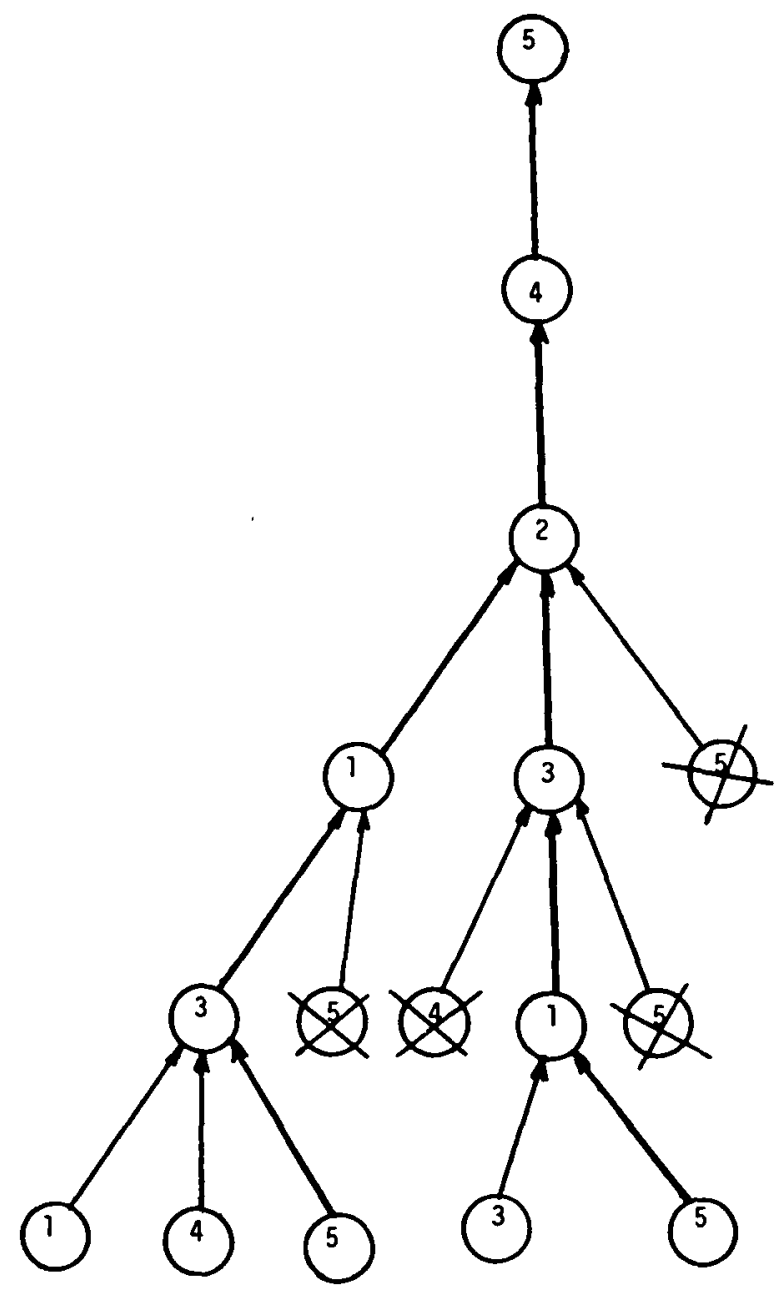

Figure 2. Detection of Hamiltonian circuits of Figure l. 
Example 2. Consider the graph in Figure 3. Here

$$
\begin{aligned}
& A=\tilde{A}_{1}=\left[\begin{array}{llll}
0 & 1 & 0 & 0 \\
1 & 0 & 1 & 0 \\
0 & 1 & 0 & 1 \\
0 & 0 & 1 & 0
\end{array}\right], \quad \tilde{A}_{3}=\left[\begin{array}{llll}
0 & 1 & 0 & 1 \\
0 & 0 & 1 & 0 \\
0 & 1 & 0 & 0 \\
1 & 0 & 1 & 0
\end{array}\right], \\
& C=\left[\begin{array}{llll}
0 & c_{12} & 0 & 0 \\
c_{21} & 0 & c_{23} & 0 \\
0 & c_{32} & 0 & c_{34} \\
0 & 0 & c_{43} & 0
\end{array}\right] .
\end{aligned}
$$

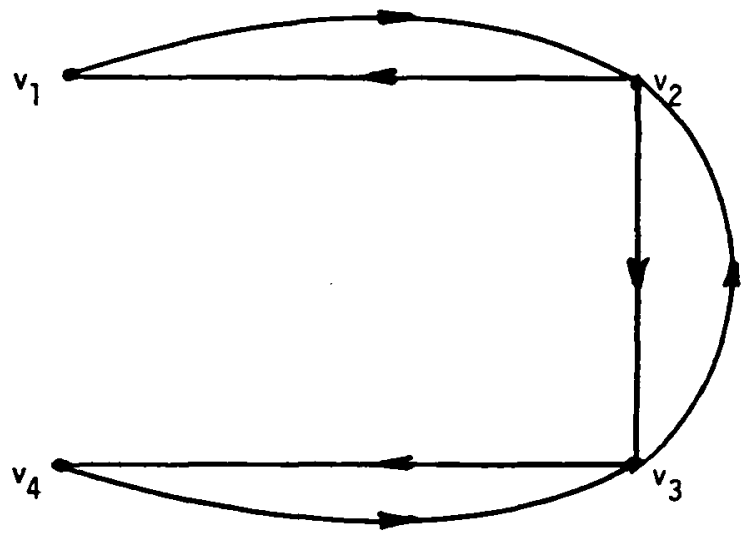

Figure 3. Graph of Example 2.

From (2),

$$
\begin{array}{ll}
d_{11}=c_{21}, & d_{22}=c_{32}, \\
d_{33}=c_{23}, & d_{44}=c_{34} .
\end{array}
$$

Since $d_{i i} \neq 0$ for all $i=1,2, \ldots, 4$, the necessary condition for the existence of a Hamiltonian circuit is satisfied. The reachability is given by

$$
v_{1} \leftarrow 2, \quad v_{2} \leftarrow 3, \quad v_{3} \leftarrow 2, \quad v_{4} \leftarrow 3
$$

Since vertices 4 and 1 do not appear in the righthand side of the above reachability no Hamiltonian circuit is possible as shown in Figure 4. 


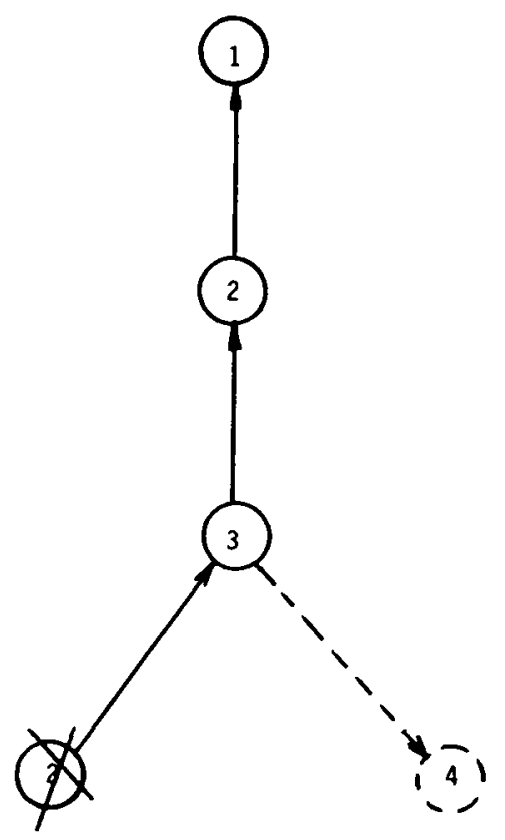

Figure 4. No Hamiltonian circuit exists in the graph of Figure 3.

Example 3. Consider the graph of Figure 5. Here,

$$
A=\tilde{A}_{1}=\left[\begin{array}{llll}
0 & 1 & 0 & 0 \\
1 & 0 & 1 & 0 \\
1 & 1 & 0 & 1 \\
0 & 0 & 0 & 0
\end{array}\right] .
$$

Since all the elements of $\tilde{A}_{1}$ in the fourth row are 0 's, no vertex can be reached from the vertex 4. Hence no Hamiltonian circuit can exist.

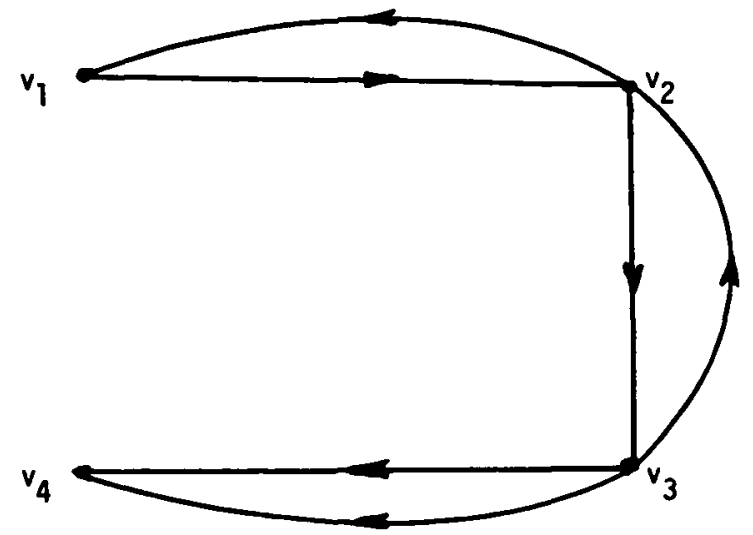

Figure 5. Graph of Example 3. 


\section{Concluding remarks}

A simple algorithm has been presented to find the necessary condition for the existence of a Hamiltonian circuit in any planar directed graph. The algorithm is based heavily on the properties of the adjacency matrix of the graph. Some obvious conclusions can be made by examining the adjacency matrix itself. If all the elements in a row or column are 0's the graph is not connected and hence no Hamiltonian circuit can exist. If all the elements are l's, the graph is complete and hence a Hamiltonian circuit must be present. Since the elements of the matrices generated can only be 0 's or 1's, the matrix multiplication can be obtained with relatively less effort by binary operation even for a matrix of large order. It is easy to program on a digital computer and by storing only the nonzero elements of the matrices computer storage can be greatly reduced. Since the product of two Boolean $n \times n$ matrices can be completed in $O\left(n^{281}\right)$ steps [1] and the algorithm requires $n$ matrix multiplications, the computational complexity of the present algorithm will be in the range of $O\left(n^{3.81}\right)$. In practice the range will be much smaller than $O\left(n^{3.81}\right)$ since in each matrix multiplication as soon as 1 has been obtained in an element, the row by column computation will be stopped. After obtaining the necessary condition, by applying the search procedure introduced in the text the existing Hamiltonian circuits can easily be traced.

\section{References}

[1] A. V. Aho, J. E. Hopcraft and J. D. Ullman, The design and analysis of computer algorthms (Addison-Wesley, Reading, Mass, 1974).

[2] R E. Bixby and D. Wang, "An algorithm for finding Hamiltonian circuits in certain graphs", Math. Programming Stud. 8 (1978), 35-49.

[3] N. Deo, Graph theory with applications to engineering and computer sctence (Prentice-Hall, Englewood Cliffs, N.J., 1974).

[4] M. R. Gary, D. S. Johnson and R. E. Tarjan, "The planar Hamiltonian circuit problems is $N P$ complete", SIAM J. Comput 5(1976), 704-714.

[5] M. S. Krishnamoorthy, "An NP-hard problem in bipartite graphs", SIGACT News 7 (1975), $1-26$.

[6] K Takamizawa, T. Nishizeki and N. Saito, "An $O\left(p^{3}\right)$ algorithm for finding Hamiltonian cycles in certain digraphs," J. Inform. Process. 3 (1980), 68-72.

[7] S. S. Yau, "Generation of all Hamiltonian circuits, paths, and centers of a graph and related problems," IEEE Trans. Circunt Theory CT-14 (1967), 79-81. 